\title{
Diabetes mellitus tipo I: retos para alcanzar un óptimo control glicémico
}

\section{Diabetes Mellitus Type I: Challenges to achieve an optimal glycemic control}

Pág. 84,95

Dra. Carolina Mejía Arens ${ }^{1}$

Dra. Nathalia Sandí Ovares ${ }^{2}$

Dra. Natalia Salazar Campos ${ }^{3}$
Recibido: 04-05-2020

Aceptado: 21-06-2020

1. Médico General, Área de Salud Zapote Catedral,Caja Costarricense del Seguro Social,Costa Rica.

2. Médico General, Hospital México, Caja Costarricense del Seguro Social,Costa Rica.

3. Médico General, Área de Salud Coronado, Caja Costarricense del Seguro Social, Costa Rica.

\section{RESUMEN}

La diabetes mellitus tipo 1 , se considera una enfermedad altamente prevalente en la población pediátrica, tiene una de etiología autoinmune compleja, que se caracteriza por la destrucción autoinmune de las células beta del páncreas, lo que conlleva a una deficiente producción de insulina, que se manifiesta clínicamente como hiperglicemia. El diagnóstico se realiza en base al cuadro clínico y hallazgos de laboratorio. El pilar de tratamiento para alcanzar un control glicémico óptimo es un esquema intensivo con insulina en dosis múltiples. A pesar de esto la mayoría de pacientes mantienen un control subóptimo de la glicemia, lo que ha llevado a investigar e identificar los posibles factores implicados en el control subóptimo de la enfermedad.

\section{PALABRAS CLAVE}

Diabetes mellitus tipo1, etiología, metas, insulina, hiperglucemia, cetosis.

\section{SUMMARY}

Type 1 diabetes mellitus is a disease, being a highly prevalent disease in the pediatric population, of complex autoimmune etiology, characterized by destruction of pancreatic beta cells in genetically predisposed individuals and the consequent deficiency in insulin production and hyperglycemia. The diagnosis is based on clinical features and laboratory findings. The key element of treatment for optimal glycemic control is an intensive multiple-dose insulin regimen. Despite this, most patients have suboptimal glycemic control, which has led to 
the investigation and identification of possible factors involved in suboptimal control of the disease.

\section{KEY WORDS}

Diabetes mellitus, type1, etiology, goals, insulin, hyperglycemia, ketosis.

\section{INTRODUCCIÓN}

La diabetes mellitus tipo 1, también llamada diabetes insulino dependiente, es una enfermedad de etiología autoinmune, que produce alteraciones en el metabolismo de los carbohidratos y lípidos (1).

Se caracteriza por la destruccion autoinune de celulas beta pancreáticas en individuos geneticamente predispuestos, alcanzando una deficiencia abdoluta en la produccion de insulina y el consecuente estado de hiperglicemia que progresa rápidamente a cetoacidosis y muerte si no es taratada con insulina $(2,3)$.

La diabetes tipo 1 sigue siendo la forma más común de diabetes mellitus en la población pediátrica y adolescente, al ser una enfermedad altamente prevalente en esta población, obliga a los padres o cuidadores, a ser los responsables de administrar la terapia, lo cuál constituye un reto para éstos y ha llevado a la implementación de nuevas tecnologías que faciliten su aplicación $(4,5,6)$.

Se han creado diversos agentes terapéuticos, como análogos de insulina recombinante, las bombas de insulina y los dispositivos nuevos para el monitoreo en el hogar, así como la investigación de terapias no insulínicas, vía oral, con el objetivo de facilitar el control de la glicemia. Sin embargo, a pesar de esto, la enfermedad mantiene un control sub óptimo y persiste cierto grado de hiperglucemia en prácticamente todos los pacientes $(7,8,9)$.

El objetivo de este artículo es describir los principales problemas implicados como posibles responsables del control subóptimo de la enfermedad, ya que representan el principal reto a corregir para alcanzar un manejo terapéutico optimo, que permita disminuir los efectos deletéreos en la salud, asociados a la persistencia de hiperglucemia a largo plazo.

\section{MÉTODO}

Para elaborar este artículo, se revisaron diferentes fuentes bibliográficas, tanto en inglés como en español, consultadas de las bases de datos de PubMed, ScienceDirect y Scielo. Los artículos seleccionados son en su mayoría revisiones sistemáticas, artículos científicos y guías de manejo actualizadas de diabetes mellitus tipo 1 en pediatría. Se eligen los 20 artículos de alto contenido científico, enfocados en el tratamiento y manejo de la enfermedad. Debido a la alta prevalencia de la DM1 en la infancia, se descartaron artículos que se basan en Diabetes Mellitus en la poblacion adulta.

\section{EPIDEMIOLOGÍA}

La diabetes mellitus tipo 1 (DM1) es una enfermedad con alta prevalencia que afecta a un número significativo de la población mundial, solo en Estados Unidos uno de cada 400 jóvenes son diagnosticados y de estos, al menos un $15-20 \%$ son menores decinco años (4). La incidencia anual ha ido en aumento entre un dos y un tres porciento, desde el 2001 al 2015. Con mayor incidencia entre los menores de 15 años y especialmente, en menores de cinco años (1, 5). 
Datos recientes revelan que muchos presentan cetoacidosis diabética (CAD) al momento del diagnóstico y mantienen un autocontrol subóptimo de la glicemia (4). De hecho, solo el 23 $\%$ de los niños de dos a cinco años cumplen las metas de glicemia establecidas por la Asociación Americana de Diabetes (HbA1C $<7,5 \%$ ). Sin una nueva estrategia que permita alcanzar las metas terapéuticas, se espera que aumenten las complicaciones a largo plazo, producto del inadecuado control sobre la glicemia en esta población (10).

\section{ETIOLOGÍA}

La DM1 es una enfermedad desencadenada por mecanismos autoinmunes, asociados en su inicio o progresión a factores ambientales y genéticos, los cuáles serán descritos a continuación

\section{ETIOLOGÍA INMUNOLÓGICA}

La diabetes mellitus tipo 1 (DM1) es una enfermedad de origen autoinmune que afecta las funciones tanto endocrinas como exocrinas del páncreas (7), donde las células presentadoras de antígeno (APC) presentan antígenos de células beta pancreáticas al sistema inmune desencadenando una respuesta inmunológica aguda, que normalmente es frenada por mecanismos de autotolerancia, pero en caso de la DM1, estos son deficientes, por lo que conducen a una respuesta inmunológica crónica que, finalmente, lleva a la destrucción de las células beta y la consecuente ausencia de secreción endógena de insulina $(6,11)$. La falla en los mecanismos de autotolerancia se ha asociado desde el punto de vista genético a polimorfismos en el gen de la proteína tirosina fosfatasa no receptora 22 (PTPN22) que codifica la tirosina fosfatasa (LYP) específica de linfocitos, un regulador negativo de la respuesta inmune (6).Este proceso se explica en el Modelo de Eisenbarth que describe el curso natural de la enfermedad, donde el evento autoinmune inicial precipitante provoca la pérdida de masa y función en las células beta. Este inicio de la autoinmunidad puede ser cuantificado por la presencia de autoanticuerpos, sin embargo, la caída de la función de las células $\beta$ no se correlaciona directamente con la disminución de la masa de las células $\beta$, ya que en algunos casos es posible detectar péptido $C$ residual en personas con DM1 de larga duración.

Las alteraciones en la glicemia ocurren incluso antes del diagnóstico clínico, ya que en muchos casos pasan desapercibidas, hasta el momento del debut diabético. Se ha cuantificado el efecto del medio ambiente en el desarrollo y progresión de DM1 y se ha descrito que este puede afectar en cualquier momento durante todo el curso de la enfermedad $(1,5)$.

En cuanto a la alteración de la función exocrina del páncreas, al disminuir las hormonas de los islotes, que tienen función reguladora sobre el tejido exocrino, pueden afectar la síntesis provocando insuficiencia exocrina, se han descrito reportes hasta en un $40 \%$ de los pacientes con DM1, de síntomas gastrointestinales como náuseas, distensión abdominal, diarrea, esteatorrea y pérdida de peso, por lo que se ha planteado que podrían asociarse a deficiencias de la función pancreática exocrina (PEI) y se sugiere investigar su presencia en caso de síntomas clínicos o control glucémico deficiente a pesar de una dieta adecuada, y adecuada adherencia al tratamiento (12).

\section{ETIOLOGÍA GENÉTICA}

El tamizaje genético es una opción clave en la identificación y diagnóstico temprano de la 
enfermedad, incluso antes del momento de la presentación clínica (2).

La DM1 tiene herencia poligénica, por lo que es posible estimar el riesgo de padecer la enfermedad para seleccionar las poblaciones por tamizar. Según la presencia de antecedentes heredo familiares, en el caso de gemelos concordantes, el riesgo de padecer DM1 si uno de los gemelos la padece es de un $30-70 \%$, el riesgo de padecerla teniendo un hermano con DM1 es de un seis a un siete por ciento y con un progenitor con DM1, es de un uno a un nueve porciento. Además, existe un patrón de comportamiento de acuerdo con el sexo, ya que es ligeramente más común en hombres y niños (5).

El mayor riesgo de desarrollar DM1 asociado a HLA se debe a la inestabilidad en la expansión en los genes que se encuentran en los loci que codifican DR y DQ de clase II, los cuales tienden a que alelos específicos en dos loci se encuentren juntos más frecuentemente de lo esperado (2). Se han descrito dos haplotipos HLA de clase 2, principalmente, implicados en la presentación del antígeno HLA DRB1 * 0301-DQA1 * 0501DQ * B10201 (DR3) y HLA DRB1 * 0401-DQA1 * 0301-DQB1 * 0301 (DR4-DQ8); en un 50\% de los pacientes con enfermedad hereditaria y son prevalentes en personas blancas (5).

En estudios europeos, en población de alto riesgo, se ha determinado un buen valor predictivo para los alelos HLA de mayor riesgo, en combinación con SNP seleccionados [119e121]. Con solo cuatro SNP, dos que etiquetan HLA (DR3 y DR4) y uno en cada uno de los genes de insulina y CTLA4, se alcanza un alto valor predictivo clínicamente relevante para predecir mayor riesgo de padecer DM1(2).

\section{FACTORES AMBIENTALES}

PRICIPALES FACTORES DESCRITOS $(5,6,11)$

- Obesidad: factor principalmente asociado, ya que se ha demostrado que el aumento de la masa de tejido adiposo causa resistencia a la insulina.

- Alteración en la microbiota intestinal: una disminución en esta puede desencadenar la activación de mecanismos autoinmunes en el tracto gastrointestinal.

- Factores dietéticos: se ha asociado al desarrollo de la enfermedad la introducción de alimentos que contienen gluten en menores de tres meses, así como la introducción temprana de fruta en la alimentación y el consumo excesivo de lácteos, por una reacción cruzada entre la insulina bovina y la humana.

- Vitamina D: en contraste con los factores anteriormente descritos, se ha demostrado que un nivel adecuado de vitamina $D$ disminuye el riesgo de diabetes, ya que el agonista del receptor de vitamina $D(V D R)$ induce a las células $T$ reguladoras (Treg) estimulando la tolerancia inmunológica.

-Virus entéricos: se han implicado las infecciones persistentes por enterovirus, rotavirus, citomegalovirus, virus de las paperas, de la rubéola, virus de Ljungan y retrovirus en la patogénesis y progresión de la DM1. Por su similitud estructural a los antígenos de las células beta, ciertas estructuras de éstos virus logran inducir la destrucción de células beta mediante mecanismos directos, citolíticos y activación indirecta autoinmune. 


\section{PRESENTACIÓN CLÍNICA}

Aunque clásicamente la DM1 se clasifica como una diabetes juvenil, esta podría ocurrir a cualquier edad, incluso un $50 \%$ de los casos se presentan en edad adulta, lo cual dificulta el diagnóstico, ya que inicialmente reciben el diagnóstico erróneo de DM2. Los síntomas de presentación más comunes en niños son los síntomas clásicos, la triada poliuria, polidipsia, y pérdida de peso, sin embargo, en muchos casos pasan desapercibidos y al menos un tercio de los casos se presenta inicialmente como cetoacidosis diabética. $(1,5)$.

\section{CRITERIOS DIAGNÓSTICO}

Para realizar el adecuado diagnóstico, debe cumplir al menos un criterio de los siguientes: $(1,5,13)$.

-Glicemia en ayunas $>126 \mathrm{mg} / \mathrm{dL}$

- Glicemia al azar en >200 mg/dL asociado a síntomas o un resultado anormal en la prueba de tolerancia oral con carga de glucosa.

- Glicemia al azar en >200 mg/dL (alterada) en al menos dos ocasiones, en ausencia de síntomas.

El diagnóstico también puede realizarse mediante valores de hemoglobina glicosilada $\mathrm{HbA} 1 \mathrm{c}>6,5 \%$, sin embargo, como la progresión de los trastornos en la regulación de la glicemia puede ser acelerada en DM1, la $\mathrm{HbA} 1 \mathrm{c}$ es menos sensible comparada con la glicemia en ayunas o la curva de tolerancia a la glucosa (1, $5,13)$.

Cabe destacar que ninguna característica clínica puede distinguir perfectamente entre la
DM1 y la no tipo 1 al momento del diagnóstico, por lo que la clasificación depende de los factores de riesgo y características clínicas, como el índice de masa corporal (IMC) y la presencia de biomarcadores (autoanticuerpos pancreáticos). Siendo estos últimos los más útiles en el diagnóstico diferencial con otros tipos de diabetes mellitus, ya que en $>90 \%$ de los diagnósticos recientes de DM1, existe presencia de anticuerpos cuantificables contra proteínas específicas de células- $\beta$, incluyendo insulina, glutamato descarboxilasa, antígeno del islote, transportador de Zn y tetraspanin-7 (5).

En estudios de cohorte, con individuos con alto riesgo genético para DM1, se ha observado una incidenciapicoeneldesarrollodeautoanticuerpos antes de los dos años. Los portadores de un solo autoanticuerpo no necesariamente progresan a DM1, pero la seroconversión a dos o más autoanticuerpos séricos se asocia a un riesgo del $84 \%$ de padecer DM1 clínica a la edad de 18 años. La concentración de péptido $C$ se puede utilizar como marcador de la deficiencia de insulina endógena severa, además, se puede utilizar para guiar la clasificación y el tratamiento $(1,5)$.

\section{METAS DE TRATAMIENTO}

La International SocietyforPediatric and Adolescent Diabetes (ISPAD) recomienda para un control glicémico óptimo un nivel de $\mathrm{HbA} 1 \mathrm{c}<7,5 \%$, siempre y cuando se garantice un control seguro sin hipoglicemia. Estudios publicados por el ISPAD 2018 demuestran que la monitorización frecuente de la glucosa junto con el ajuste óptimo de la insulina, según la ingesta de carbohidratos y el ejercicio, son necesarios para lograr y mantener este control metabólico óptimo $(4,13)$.

\section{(c) $\underset{\text { BY }}{\ominus} \underset{\mathrm{ND}}{\mathrm{NC}}$}


Diversos estudios demuestran que alcanzar y mantener glicemias cercanas a las observadas en personas sin diabetes reduce las complicaciones micro y macro vasculares en DM1, para alcanzar estas metas, se recomienda la monitorización de $\mathrm{HbA} 1 \mathrm{c}$ de cuatro a seis veces al año en niños pequeños y de tres a cuatro veces en niños mayores $(4,7,13)$.

El desafío en el manejo adecuado de los niveles de glicemia, a pesar de contar con medicamentos aprobados por la agencia responsable de la regulacon de alimentos y medicamentos de los Estados Unidos, FDA (Food and Drug Administration: Administración de Alimentos y Medicamentos) para el manejo, se ha atribuido a diversas causas, como que, durante la primera infancia, son los padres quienes están a cargo de realizar tareas complejas de manejo, como administración de insulina, cálculos de dosis, control de glicemia y planificación de comidas. Sumado a esto, existen variaciones fisiológicas en las necesidades de insulina, variaciones en el grado de sensibilidad a la insulina, variaciones en el grado de desarrollo cognitivo, conductual y social de los niños pequeños, así como escasez de recursos en algunos casos. Todas estas variantes representan verdaderos obstáculos para mantener un adecuado monitoreo y control óptimo de la glicemia $(4,10)$.

Un aspecto poco estudiado, en cuanto a los retos en el manejo de la DM1, es la angustia de los padres o cuidadores ante las crisis de hipoglicemia; estos sentimientos pueden llevar a comportamientos como el sobretratamiento de la hipoglucemia, la administración de insulina por debajo de la dosis recomendada, para mantener un nivel de glucosa en sangre superior al recomendado o iniciar el tratamiento de la hipoglucemia cuando los niveles de glucosa están por encima del rango de hipoglucemia (70 mg / dl) (10). Por lo que Berget et al.,(10) en su estudio proponen intervenciones conductuales y educación para los padres, ya que estas intervenciones tienen el potencial de reducir el miedo a la hipoglucemia, con el fin de alcanzar un control de la glicemia más satisfactorio.

En adolescentes con DM1, es necesario, además, cambiar gradualmente las tareas de inyección de insulina y monitoreo de glicemia de sus padres a ellos mismos. Su adaptación a este cambio es crucial en su bienestar a largo plazo y se ha demostrado una mejor calidad de vida si desarrollan independencia y autoeficacia en estas tareas. La Escala de autocontrol de la diabetes percibida (PDSMS) se utiliza para medir la confianza de los pacientes en el manejo adecuado de su control glucémico (autoeficacia). PDSMS demostró que una mejor autoeficacia (puntajes más altos) se asocia con un mejor control glucémico (nivel de HbA1c más bajo) (14).

Aunque la Asociación Estadounidense de Diabetes ha recomendado un estándar menos estricto $(<7,5 \%)$ para adolescentes y adultos jóvenes de 13 a 19 años, ha enfatizado que una meta de $<7 \%$ es razonable si se puede lograr sin hipoglucemia (14). Ante este reto, se ha planteado combinar la terapia con educación y orientación intensiva a los padres y cuidadores, que les brinde herramientas para no solo detectar las variaciones en los niveles de glicemia a lo largo del día, sino también prepararlos para realizar ajustes adecuados de dosis según la necesidad (4).

La monitorización continua de glucosa (MCG) es importante debido a su capacidad para mostrar la variabilidad de la glucemia y la hipoglucemia no reconocida, permitiendo una mayor precisión y flexibilidad en la dosificación de insulina y 
detección rápida de fluctuaciones de glucosa, así como las opciones predictivas para el control metabólico a largo plazo, se recomienda su uso en todos los pacientes con DM1 al menos una vez al año. Además, al permitir la detección de grandes fluctuaciones en la glucemia, puede utilizarse como ayuda en la corrección adecuada de la terapia. Sin embargo, el problema sigue siendo la gran cantidad de inyecciones y la saturación del sitio de aplicación, lo que complica la regulación de la diabetes y reduce la calidad de vida $(8,9,10)$.

\section{OPCIONES DE TRAMIENTO}

\section{TRATAMIENTOS INSULÍNICOS}

La insulina sigue siendo el pilar de la terapia, la insulina NPH (protamina neutra de Hagedorn) se utiliza con más frecuencia en menores de cinco años, en combinación con análogos rápidos postprandiales y en menor frecuencia, insulina regular preprandial; esta produce una $\mathrm{HbA1c}$ más favorable, pero con mayor riesgo de hipoglicemia. Según ISPAD 2018, el uso de análogos de acción prolongada en comparación con $\mathrm{NPH}$ ha mostrado una reducción en la hipoglicemia, pero no en la $\mathrm{HbA} 1 \mathrm{c}(4,13)$.

Se ha sugerido como ideal el tratamiento intensivo con insulina, en regímenes de dosis múltiples logra alcanzar el control glicémico óptimo y retrasar la aparición y progresión de la retinopatía, nefropatía y neuropatía diabética en pacientes con DM1, por lo que los nuevos análogos de insulina con diferentes inicios y duraciones de acción permiten esquemas de dosificación que se basan en aplicar una insulina basal para control nocturno (que cubre los periodos entre comidas) y varias dosis en bolo de análogos de insulina de acción rápida para cubrir las cargas de carbohidratos postpandiales y para tratar la hiperglucemia $(7,14,15)$.

En el caso de las nuevas insulinas, se ha demostrado que Aspart ultrarrápida presenta propiedades farmacocinéticas y farmacodinámicas, similares en sujetos de edad avanzada y jóvenes con DM1, por lo que son útiles para mejorar el control de glucosa posprandial. Presentan un inicio más rápido y mayor efecto en reducción temprana de la glucosa, imitando mejor la liberación fisiológica de insulina postprandial y proporcionando una mejor cobertura a la hora de comer $(14,15,16)$.

El uso adecuado de insulina requiere ajustes de dosis frecuentes según la cantidad de carbohidratos ingeridos, la actividad física y la enfermedad 0 el estrés, de manera que no afecte el desarrollo adecuado del niño en crecimiento, por lo tanto, el automonitoreo de la glicemia resulta beneficioso, solo si los padres o cuidadores están capacitados para reaccionar adecuadamente a los niveles de glicemia (7).

Para disminuir la dificultad en la aplicación de las terapias inyectadas, se crean nuevos dispositivos como la bomba de insulina (IP), sumamente útil y altamente sofisticada, que permite el suministro subcutáneo continuo de insulina de forma más similar a su secreción fisiológica, eliminando la necesidad de utilizar inyecciones. Debido a la buena regulación a largo plazo en pacientes pediátricos y la eliminación de inyecciones, el uso de bombas de insulina en niños y jóvenes en el mundo está en notable expansión. Los cambios en el flujo basal, así como el número deseado de dosis de bolo aplicadas a un niño no inyectado, permiten un monitoreo más adecuado de los requerimientos diarios variables de insulina $y$, por lo tanto, un 
control glucémico más estable $(8,9)$.

La introducción de agentes terapéuticos modernos como los análogos de insulina y la bomba de insulina (IP) ha contribuido a mejorar el control metabólico de la DM1, alcanzando niveles de $\mathrm{HbA} 1 \mathrm{c}$ hasta en un 0,4 a $0,6 \%$ menores, sin una mayor incidencia de hipoglucemia. Otros dispositivos como el PEN (lápiz de insulina), que son dispositivos con agujas de menor tamaño, con cartuchos reemplazables, se han creado con la premisa de facilitar la aplicación de insulina inyectada, en estos se ha observado una disminución en frecuencia de hipoglicemias severas, sin embargo, su efecto en la frecuencia de hipoglucemia en general, en pacientes con IP y PEN, no está clara $(1,8,9)$.

\section{TRATAMIENTOS NO INSULÍNICOS}

-Pramlintide: el único tratamiento complementario oral no insulínico aprobado para control glicémico en pacientes con DM1. Otros hipoglicemiantes orales se utilizan de forma off label, como metformina, tiazolidinedionas, análogos de amilina, inhibidores del cotransportador de sodio-glucosa 2 (SGLT2), agentes basados en incretina como agonistas del receptor del péptido similar al glucagón-1 (GLP-1) y dipeptidil peptidasa-4 (DPP-4) (11), sin embargo, menos del diez por ciento de los pacientes usa estos medicamentos. También está aprobada la terapia con glucagón para DM1, pero solo se ha comercializado como botiquín de emergencia para hipoglucemia grave que provoca convulsiones o pérdida del conocimiento (7).

-Metformina: posee efectos pleiotrópicos, en la práctica clínica, se utiliza principalmente para disminuir la resistencia a la insulina. Sin embargo, se han reportado otros mecanismos de acción interesantes, incluido su efecto protector sobre las células $\beta$ y prevención de complicaciones cardiovasculares (11).

-Inhibidores de SGLT-2: bloquean la reabsorción de glucosa en los túbulos renales, aumentando la excreción urinaria, mejoran el metabolismo de la glucosa, tienen un efecto positivo sobre el perfil lipídico y protección y regeneración de las células $\beta$ en DM 1. Algunos datos indican un papel potencial en la protección de las funciones renales, reducción de peso y presión arterial $(11,17)$.

-Amilina (polipéptidoamiloide de islotes IAPP): es una hormona anorexígena, que participa en la regulación de la secreción de insulina y glucagón, en DM1, existe un déficit de esta, lo que propicia mayor riesgo de hipoglucemia grave posterior a la insulina. Se ha visto que la terapia de infusión continua subcutánea de insulina con adición de pramlintida disminuye los niveles de $\mathrm{HbA} 1 \mathrm{c}$, masa corporal y necesidades de insulina (6).

-Agentes basados en incretinas: incluyen a los agonistas del receptor del péptido 1 similar al glucagón (GLP-1) e inhibidores de la dipeptidil peptidasa-4 (DPP-4), cuyo mecanismo de acción final es aumentar la secreción de GLP-1, que actúa estimulando la secreción de insulina, bloquea la producción de glucagón e inhibe la apoptosis de las células $\beta$ pancreáticas $(11,17)$.

\section{NUEVAS TERAPIAS}

Ha medida que ha avanzado la ciencia, se han logrado desarrollar nuevas terapias, tales como:

-Alotrasplante de islotes: se utilizan como alternativa terapéutica en pacientes con DM1 de difícil control, existen varios tipos de procedimientos, el trasplante de islotes solo, el trasplante de islote después de riñón y trasplante 
simultáneo de islote-riñón. Estos dos últimos se consideran en pacientes con insuficiencia renal (uremia). El trasplante de islote después de riñón tiene la ventaja de que ya los pacientes están inmunosupresosde por vida (18). Existen pocos estudios, con evidencia limitada, los cuales demuestran que, en pacientes con DM1 con y sin enfermedad renal, mejora el control glucémico y las complicaciones diabéticas, a pesar de que los eventos adversos aumentan en comparación con la terapia intensiva con insulina. La principal limitante para su uso es la escasez de donantes $(19,20)$.

- Células madre mesenquimales(BM-MSC): son células adultas multipotentes derivadas de médula ósea, hígado o tejido adiposo, las cuales tienen el potencial de diferenciarse en células productoras de insulina (IPC). Presnetan varias ventajas respecto a las células madre embrionarias, por su alta capacidad de diferenciación, mayor estabilidad cromosómica, menor tendencia a formar neoplasmas en el receptor y no presentan problemas de inmunocompatibilidad porque tienen baja expresión HLA-I y no expresan HLA-II, tienen un bajo riesgo de rechazoalogénicoy deenfermedad de injerto contra huésped. En estudios en ratas diabéticas, se logró documentar regeneración de páncreas endocrino (aumento en el tamaño y número de células en los islotes) después del trasplante de células productoras de insulina (IPC) $(18,20)$.

\section{COMPLICACIONES DIABÉTICAS}

Las complicaciones macrovasculares incluyen enfermedades de grandes vasos sanguíneos, como enfermedad coronaria, cerebrovascular y vascular periférica, causadas principalmente por aterosclerosis y trombosis (6). La nefropatía diabética tiene el potencial de aumentar progresivamente el riesgo de otras complicaciones macrovasculares; pero la enfermedad cardiovascular sigue siendo la principal causa de morbilidad y mortalidad prematura y su riesgo no disminuye de forma tan significativa con un control intensivo (5).

El daño a los vasos pequeños (capilares) debido a la hiperglicemia puede causar complicaciones microvasculares en los tejidos donde la absorción de glucosa es independiente de la insulina, como en las neuronas, riñones y retina. Presentándose retinopatía diabética, ocasionada por daños en mácula o retina que pueden causar problemas visuales y ceguera, la neuropatía diabética, por lesiones en nervios periféricos, incluidas las neuronas sensoriales, autónomas y motoras, así como la nefropatía diabética complicación que se detecta en aproximadamente un 30 a $40 \%$ de los diabéticos tipo 1 y se caracteriza por la pérdida de la tasa de filtración glomerular, albuminuria (> $300 \mathrm{mg} /$ día) y el daño a los glomérulos (6).

La hipoglicemia y la cetoacidosis diabética (CAD) son complicaciones agudas bastante frecuentes y potencialmente mortales. Los eventos hipoglicémicos graves incluyen aquellos que requieren asistencia terapéutica de otra persona, pérdida de la conciencia o convulsiones y se asocian a efectos adversos crónicos y agudos en la función cognitiva, que incluyen disminución de la función cognitiva, atención y percepción visual, además, se asocian a un cuatro a diez porciento de las muertes relacionadas con DM1 $(3,5)$.

La CAD se define como la presencia de hiperglucemia (glicemia $>200 \mathrm{mg} / \mathrm{dL}$ ), $\mathrm{pH}<7,3$ o bicarbonato $<15 \mathrm{mmol} / \mathrm{L}$, cetonemia y cetonuria. Los signos clínicos incluyen deshidratación, taquicardia, taquipnea, aliento a acetonas, náuseas, vómitos, confusión, somnolencia y disminución del nivel de conciencia. Es una complicación frecuente, por lo que resulta vital diagnosticarla a tiempo e incluso prevenirla.

\section{(c) (1)}


Entre los factores de riesgo para CAD se encuentran pacientes de reciente diagnóstico en especial <2 años, diagnóstico tardío, bajo nivel socioeconómico y países con baja prevalencia de DM1 $(3,13)$.

Los niños pequeños con diabetes de inicio temprano se presentan con mayor frecuencia con CAD (más de dos tercios) y los que presentan hiperglucemia presentan previo al diagnóstico síntomas clásicos evidentes y útiles en el reconocimiento y diagnóstico temprano $(1,4)$.

La causa más común de reingreso hospitalario después del diagnóstico es la CAD o hiperglucemia. Estas se deben principalmente a la omisión de dosis de insulina, control metabólico deficiente, episodios previos de $C A D$, gastroenteritis con vómitos persistentes e incapacidad para mantener la hidratación, trastornos psiquiátricos, circunstancias sociales y familiares desafiantes, época puberal y preadolescentes, acceso limitado a servicios médicos, así como fallas en la terapia con bomba de insulina $(3,4)$.

Otra de las complicaciones más frecuentes incluye el estado hiperosmolar $(E H H)$, cuyos criterios diagnósticos incluyen: glicemia $>600 \mathrm{mg} /$ $\mathrm{dL}, \mathrm{pH}>7,30$, bicarbonato $>15 \mathrm{mmol} / \mathrm{L}$, cetonuria, osmolaridad sérica $>320 \mathrm{mOsm} / \mathrm{kg}$, alteración del estado de conciencia o convulsiones (3). La hipoglucemia grave repetida, especialmente en niños pequeños, puede causar secuelas como trastornos psicomotores permanentes del desarrollo (8).

\section{CONCLUSIONES}

En la revisión bibliográfica realizada se logran identificar como principales retos a mejorar en el abordaje terapeutico para alcanzar un control óptimo de la glicemia en diabetes mellitus de tipo I, la detección tardía de la enfermedad, los factores psicosociales de los padres o cuidadores que dificultan en la aplicación de tratamientos intensivos con insulina, tales como el miedo a la hipoglicemia, o la carencia de educación suficente para realizar un ajuste de dosis según las necesidades específicas, y la inexistencia de un tratamiento modificador del curso natural de la enfermedad.

El tamizaje mediante pruebas genéticas $y$ serologías inmunes en poblaciones de riesgo, es una herramienta cada vez más disponible para la detección temprana de la enfermedad.

Se propone realizar intervenciones conductuales, y de capacitación a los padres, para que consigan llevar a cabo un adecuado monitoreo de la glicemia y puedan responder apropiadamente a los cambios en esta, con ajustes de dosis de insulina, según la necesidad. Los intentos por mejorar el control glicémico requieren realizar una adecuada transición de padres a hijos, de las tareas de inyección de insulina y monitoreo de glicemia durante la adolescencia, reforzarndo la confianza y autoeficacia en el individuo afectado.

Las nuevas terapias como adyuvantes orales, el uso de bombas de monitoreo continuo de glucosa y los lápices de insulina de aplicación subcutánea, constituyen una estrategia vital para el tratamiento, ya que disminuyen número de inyecciones necesarias, el dolor asociado a la aplicación del tratamiento y la saturación del sitio de aplicación.

El campo de investigacion a futuro está dirigido a buscar nuevas terapias que permitan regenerar la función de las células b del páncreas, para cambiar el curso natural de la enfermedad, como es el caso del transplante de células beta y el uso de células madre. 


\section{BIBLIOGRAFÍA}

1. Cooke D.W and Plotnick L. (2008).Type 1 Diabetes Mellitus in Pediatrics. Pediatr. Rev, 29;374385.

2. Noble JA. (2015). Immunogenetics of type 1 diabetes: A comprehensive review. Journal of Autoimmunity; 64: 101-112.

3. Wolfsdorf, Jl. Allgrove, J. Craig, ME. Edge J, Glaser N, Jain V, et.al. (2014). ISPAD Clinical Practice Consensus Guidelines 2014 Compendium Diabetic ketoacidosis and hyperglycemic hyperosmolar state. Pediatric Diabetes.15(S20): 154-179.

4. Mamdouh Hassan M, Arafa N, Abdou M, Hussein O. (2020) Characteristics of diabetes diagnosis and control in toddlers and preschoolers from families with limited resources: A single center experience. Diabetes research and clinical practice.159:107966.

5. DiMeglio LA., Evans-Molina. C, Oram, RA. (2018). Type 1 diabetes. The Lancet. 391(10138): 2449-2462.

6. Saberzadeh-Ardestani B, Karamzadeh R, Basiri M, Hajizadeh-Saffar E, Farhadi A, Shapiro AMJ, et al. (2018). Type 1 Diabetes Mellitus: Cellular and Molecular Pathophysiology at A Glance. Cell J. 20(3): 294-301.

7. Beck JK, Cogen FR. (2015). Outpatient Management of Pediatric Type 1 Diabetes. The Journal of Pediatric Pharmacology and Therapeutics. 20(5): 344-357.

8. Hasanbegovic S, Obarcanin E, Hasanbegovic E, Begic N. (2017). Impact of Insulin Delivery Method on Hypoglycemia Incidence in Pediatric Type 1 Diabetes Mellitus Patients. MedArch. 71(6): 391-395.

9. Langendam M, Luijf Y, Hooft L, DeVries J, Mudde A, Scholten R. (2012). Continuous glucose monitoring systems for type 1 diabetes mellitus. Cochrane Database Syst Rev. 18(1): 1-143.

10. Berget C, Driscoll K, Lagges A, Lange S, DiMeglio L, Hannon T, et al. (2019). Optimizing the use of continuous glucose monitoring in young children with type 1 diabetes with an adaptive study design and multiple randomizations, for the Toddler Diabetes Technology Study Group. Contemporary Clinical Trials. 82: 60-65.

11. Otto-Buczkowska E, Jainta N. (2018). Pharmacological Treatment in Diabetes Mellitus Type 1 - Insulin and What Else?. Int J EndocrinolMetab. 16(1): e13008. 
12. Zsori G, D Illes D, Terzin V, Ivany E, Czako L. (2018). Exocrine pancreatic insufficiency in type 1 and type 2 diabetes mellitus: do we need to treat it? A systematic review. Pancreatology. 18(5): 559-565.

13. Codner E, Acerini CL, Craig ME, Hofer SE, Maahs DM. (2018). ISPAD Clinical Practice Consensus Guidelines 2018: What is new in diabetes care? Pediatric Diabetes.19(S27): 5-6.

14. Chih AH, Jan CF, Shu SG, Lue BH. (2010). Self-efficacy Affects Blood Sugar Control Among Adolescents With Type I Diabetes Mellitus. Journal of the Formosan Medical Association. 109(7): 503-510.

15. Bolli G. (2007). The benefits of insulin analogues in intensive treatment of type 1 diabetes mellitus. Av Diabetol. 23 (5):326-332.

16. Heise T, Ho velmann U, Zijlstra E, Stender-Petersen K, Jacobsen JB, Haahr H. (2017). A Comparison of Pharmacokinetic and Pharmacodynamic Properties Between Faster-Acting Insulin Aspart and Insulin Aspart in Elderly Subjects with Type 1 Diabetes Mellitus. Drugs Aging. DrugsAging. 34: 29-38.

17. Garg V. (2011). Noninsulin pharmacological management of type 1 diabetes mellitus. Indian J Endocr Metab.15:S5-11.

18. Domouky AM, Hegab AS, Al-Shahat A, Raafat N. (2017). Mesenchymal stem cells and differentiated insulin producing cells are new horizons for pancreatic regeneration in type I diabetes mellitus. International Journal of Biochemistry \& CellBiology. 87: 77-85.

19. Health Quality Ontario. (2015). Pancreas islet transplantation for patients with type 1 diabetes mellitus: a clinical evidence review. OntHealthTechnolAssess Ser.15(16): 1-84.

20. Amer MG, Embaby AS, Karam RA, Amer MG. (2018). Role of adipose tissue derived stem cells differentiated into insulin producing cells in the treatment of type I diabetes mellitus. Gene. 654: 87-94. 